\title{
On the Accuracy of RFID-based Localization in a Mobile Wireless Network Testbed
}

\author{
Vikram P. Munishwar, Shailendra Singh, Xiaoshuang Wang, Christopher Mitchell ${ }^{\dagger}$, \\ Kartik Gopalan, Nael B. Abu-Ghazaleh \\ Computer Science, Binghamton University (State University of New York) \\ ${ }^{\dagger} \mathrm{S} *$ ProCom ${ }^{2}$, The Cooper Union for the Advancement of Science and Art \\ Contact: $\{$ kartik,nael $\} @$ cs.binghamton.edu
}

\begin{abstract}
The extensive recent research in protocols development for wireless networks must be complemented with simple yet efficient prototyping and evaluation mechanism. MiNT-2 is a miniaturized multi-hop wireless network testbed that addresses this challenge by combining the benefits of existing protocol evaluations strategies: simulations and custom built large scale wireless network testbeds. A key component of MiNT-2 is the ability of testbed nodes to move around in the testbed space, whether to reconfigure the network topology or to emulate node mobility patterns. Consequently, we need to devise accurate mechanisms for node localization - the ability of a node to be aware of its exact location in the testbed space. This paper presents the details of the current RFID-based node localization mechanism in MiNT-2. We describe the sources of inaccuracy encountered when using RFID tags due to the error margin associated with the reading range of tags. We then present the implementation and evaluation of two approaches which can be combined to improve the overall accuracy of the RFID-based localization system. Through our experimental evaluation, we show that the proposed approaches improve the accuracy of RFID-based localization mechanisms by up to $58.22 \%$ for dense deployment of RFID tags. Though described in the context of the MiNT-2 testbed, our localization techniques are also applicable in more general settings where accurate and inexpensive positioning of mobile nodes is important.
\end{abstract}

\section{INTRODUCTION}

As research and development in wireless networking protocols gains importance, it is ever more essential to carefully test the performance of protocols under situations closely resembling the real world. Two currently predominant evaluation mechanisms are software simulations and large-scale testbeds, Although simulations [1]-[3] offer control and repeatability of experiments, they do not reflect the real-world radio propagation effects such as non-uniform path loss, multi-radio interference, multi-path fading, etc. On the other hand, custombuilt large-scale testbeds [4]-[6] accurately reflect the timeand space-varying characteristics of RF propagation, but incur large setup costs, offer limited reconfigurability to reflect a range of experimental scenarios for evaluation, and require extensive manual maintenance.

In the MiNT-2 project [7], we aim to combine the convenience of software simulations with the realism of realworld testbeds. There are two central architectural features of the MiNT-2 testbed. First is support for node mobility and reconfiguration of network topology through the use of commodity iRobot Create robots. Second is the miniaturization of the physical space required for the testbed by shrinking each node's wireless transmission range through the use of radio signal attenuators [8] and transmission power control. This paper focuses on the enabling technology behind the first feature - node mobility and testbed reconfigurability.

The ability of the nodes to move around in the testbed space enables the user to reconfigure the network topology and to specify various node mobility patterns so as to evaluate network protocols under a number of different scenarios. The effectiveness of this capability is dependent on the accuracy of the node localization mechanism, that is, the ability to accurately pinpoint the position of each node within the testbed space. Thus accurate localization of nodes is one of the fundamental requirements of the MiNT-2 testbed.

The design goal of our localization technique is to achieve a high level of accuracy while at the same time keeping the cost of localization hardware low. The iRobot Create already comes with two built-in sensors - for distance and angle measurements - to track the robot's movement. These sensors can be queried via the serial interface, and return both the distance traveled by the robot and angle the robot has rotated through since the last query. In principle, if the initial position of the robot is known accurately, the data returned by these two sensors should be sufficient for tracking the position of the robot as it moves through the testbed space. In practice, however, the accumulated error in distance and angle measurements grows over time due to a number of factors such as rounding errors, wheel slippage, and encoder inaccuracy. Furthermore, a node might be manually picked up and moved to a new location. Thus we cannot entirely rely on internal sensor readings from the robot. Rather we need additional mechanisms to perform accurate localization over time.

The MiNT-2 testbed employs a Radio Frequency Identification (RFID) tag-based mechanism for node localization. An RFID tag consists of an integrated circuit and a radio antenna, that can be used to store a unique identification identifier and is capable of returning this information in response to a radiofrequency (RF) signal emitted by an RFID reader device. RFID tags can be either active (containing a battery), or passive (without a battery). Passive RFID tags, in particular, use the external RF signal emitted from an RFID reader device to induce a minute electrical current in their antenna, which is just sufficient for the integrated circuit in the tag to transmit 


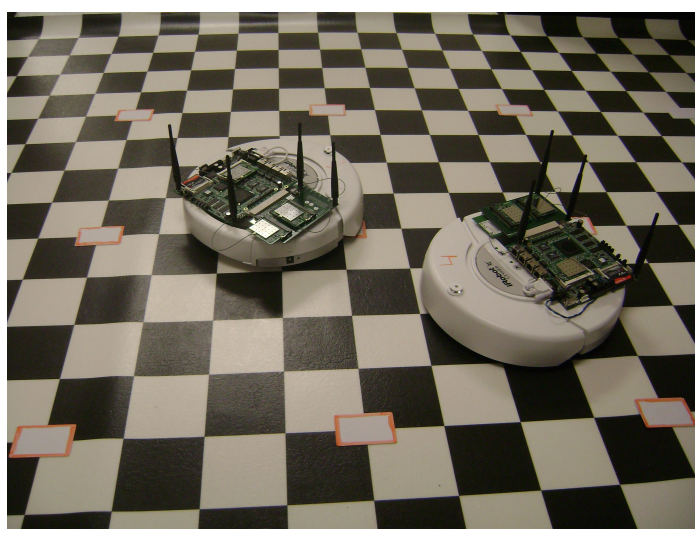

Fig. 1. Picture of the MiNT-2 testbed. RFID cards are marked red.

the stored unique identification value. As a result, compared to active tags, passive RFID tags also tend to have a smaller range within which their values can be read. This property of passive tags turns out to be very well-suited for the localization mechanism in the MiNT-2 testbed.

A rudimentary algorithm for RFID-based localization in MiNT-2 was described in our earlier work [7]. The basic idea is to deploy a grid of RFID tags on the floor area of the testbed and assign an $(x, y)$ coordinate to each tag value in the testbed area. Thus, when a robot, carrying an RFID reader, passes over an RFID tag, it calibrates its position by assigning the coordinate of that tag to itself. Between two tags, the robot uses its internal angle and distance sensors to estimate its position. This hybrid approach, while simple and more accurate than using sensors values alone, still suffers from nonnegligible inaccuracies. These inaccuracies arise from several sources, including the fact that the RFID tag value can be sensed by the reader device within a circle of radius $2.25 \mathrm{~cm}$. This, when coupled with the inherent inaccuracy of the robot's internal angle sensors, leads to significant inaccuracy in position estimates over time. We describe the details of our earlier localization technique and its limitations in Section II.

In this paper, we propose two schemes to improve the accuracy of RFID-based localization mechanism in MiNT-2 by allowing nodes to refine their position upon detection of multiple RFID tags over a straight line movement, and adaptively choosing accurate position estimate from RFID- and sensor-based readings. We describe the proposed mechanisms in Section III and Section IV and their evaluation in Section V. The related work is presented in Section VI, and conclusions along with directions for future work are given in Section VII.

\section{BACKGROUND AND MOTIVATION}

In this section, we provide a brief overview of the MiNT-2 architecture and earlier RFID-based localization mechanism, and subsequently elaborate on problems with earlier approach.

[Overview of MiNT-2 ] The main components contributing to the new design of the MiNT-2 node are the iRobot Create platform to achieve node mobility and the Soekris net5501 x86 embedded board (with multiple wireless cards attached) to run

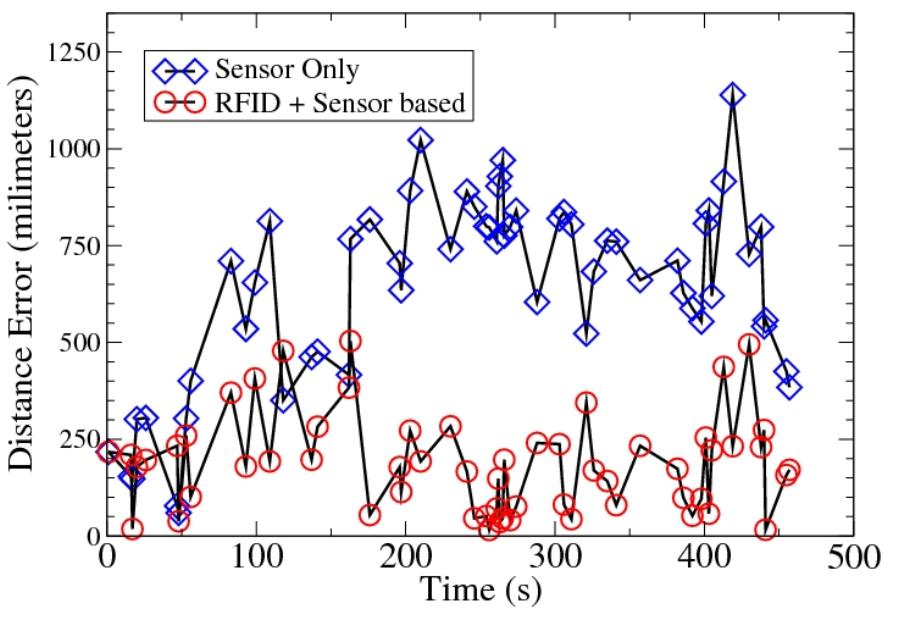

Fig. 2. Errors in sensor-only vs. RFID+sensor-based localization with time.

networking applications, simulation packages (e.g. NS2), and applications to control movements of the robot. For automatic recharging of the node's battery, Create robots are designed to use docking stations. Each node has at least two wireless communication interfaces: one to exchange control packets and one or more to exchange data packets. The wireless interfaces used in the node design are Routerboard R52 802.11a/b/g cards based on the Atheros AR5414 chipset. Each wireless interface is attached to an external antenna through a fixed attenuator, in order to miniaturize the radio communication range. A low frequency RFID reader $(125 \mathrm{~K} \mathrm{~Hz})$ is also attached to each node in order to be able to localize itself upon detection of an RFID tag. Figure 1 presents the details of the MiNT-2 node and testbed design.

The key features offered by the MiNT-2 testbed include: miniaturization of the physical space by attenuating the radio signals, support for automatic reconfiguration and management, support for testing robustness of protocols by using network fault injection, and compliance with the existing NS2 based simulation code.

[MiNT-2 Localization Mechanism] The earlier localization mechanism [7] enables a node to re-calibrate its position using the coordinates of the detected RFID tag. To enable position estimation between successive detection of two tags, the builtin distance and angle sensors of robots are used. While a robot's position calibration can be performed upon detection of a single RFID tag (just by assigning the $(x, y)$ coordinates of the tag to itself), its orientation calibration needs the positions of at least two RFID tags that are traversed in a straight line movement. Essentially, if $(x 1, y 1)$ and $(x 2, y 2)$ are the coordinates of the two tags encountered in a straight line, the robot's orientation can be calculated as follows:

$$
\theta=\left(\tan ^{-1}\left(\frac{y_{2}-y_{1}}{x_{2}-x_{1}}\right)+\frac{\Delta \theta_{R F I D}}{2}\right) \% 360
$$

where the term $\frac{\Delta \theta_{R F I D}}{2}$ is used to account for constant radius arc movement of the robot. Thus, when a robot is placed anywhere in the testbed, it needs to travel in a straight 


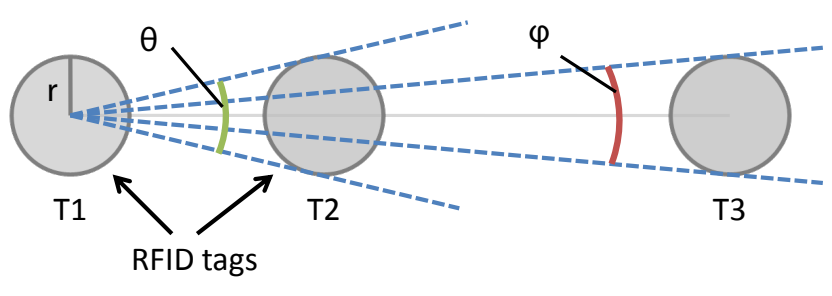

Fig. 3. The figure shows that the maximum error in angle using closely spaced tags T1 and T2 $(\theta)$ is greater than the maximum error in angle using farther tags $\mathrm{T} 1$ and $\mathrm{T} 3(\phi)$.

line until it crosses two RFID tags, to initialize its position and orientation (we term this as an initialization process in the paper). Figure 2 shows the effectiveness of RFID-based localization over one purely based on robot's in-built sensors.

As can be seen from the figure, although RFID-based localization clearly outperforms the pure sensors based localization, the average error obtained through RFID-based mechanism is still approximately $25 \mathrm{~cm}$, which is a fairly large error value, especially for its applications in automatic topology formation based on the given coordinates information of nodes, robot collision avoidance, etc. The reason behind this error value can also be conjectured as the error in orientation calibration, since the maximum error in position calibration is limited by the uncertainty in the RFID-tag reading range $(2.25 \mathrm{~cm})$.

\section{RFID-BASED CONTINUOUS REFINEMENT APPROACH (RCRA)}

Two factors affecting the accuracy of RFID tags based orientation calibration mechanism are the reading range of each RFID tag and the density of tags deployment. Since, the reading range of a tag is constant in our case - a circle of $2.25 \mathrm{~cm}$ radius - the deployment density of RFID tags is the only parameter that can be adjusted to improve the accuracy. Figure 3 shows the relationship between tags density and maximum error bound in orientation calibration. If two consecutive tags encountered by the robot when travelling in a straight line are close to each other, then the resulting uncertainty in orientation is $\theta$ at the second tag. If the two consecutive tags are further apart, then the resulting uncertainty is $\varphi$. Since $\varphi<\theta$, the farther the consecutive tags are, the better will be the orientation accuracy.

However, while increasing the distance between tags may reduce the orientation calibration, it may increase the overall error in robot's position estimate. More specifically, lower density of RFID tags increases the average time interval between successive detection of two RFID tags. As a result, the error in position estimates purely based on sensor values starts accumulating as shown in the Figure 2.

To address this problem, we use a continuous refinement based approach for orientation calibration. Essentially, we neither reduce the tag density nor calibrate the orientation at every two successive detections of RFID tags. Instead, the robot calibrates its orientation using the coordinates of the first and the latest tag encountered on a straight line movement. Thus, as long as the robot travels in a straight line, it can successively refine its orientation estimate with each tag encountered on its path, without compromising the accuracy of its position estimate.

\section{Adaptive Localization Scheme (ALS)}

RCRA continuously improves the orientation estimates when the robot travels in a straight line. However, if the robot makes frequent turns or encounters obstacles (such as other robots), the orientation refinement process would be reset at each turn, resulting in larger error bounds of the magnitude seen with the scheme described in Section II. The resulting error could be even greater than the maximum error observed for with purely sensors-based estimation.

To address this general situation, when the robot could often turn or encounter obstacles, we propose to adaptively select between the RFID based calibration and the sensors based calibration, depending on the maximum error bound offered by the schemes. Essentially, there needs to be a lower bound on the number of RFID tags to be used for orientation-calibration, based on the tags' deployment density. If the maximum number of tags detected during a straight line movement is less than this threshold, robot can fall back to the sensors based orientation calibration. Please note that we deploy the RFID tags uniformly in the testbed area, thus the lower bound in terms of number of tags is the same as the lower bound on the distance between two RFID tags encountered along a straight line path.

Since the reading range of RFID tags is constant, we can estimate the maximum error bound using RFID based orientation-calibration for a given deployment scenario as:

$$
\text { error }_{\max }=2 * \sin ^{-1}\left(\frac{r}{d}\right)
$$

where, $r$ is the radius of the reading range circle of an RFID tag, and $d$ is the distance between two RFID tags. However, since the error in sensors based estimations is not dependent on the constant or predictable parameters (it can occur due to rounding errors, wheel slippage, and encoder inaccuracy), it is difficult to choose between the sensors and RFID based estimations at the run-time.

In order to address this problem, we propose to use a static method to calculate the lower bound on the number of RFID tags to be considered for calibration. Essentially, we perform a measurements based study, in which each experiment is performed for a fixed value of the lower bound on number of tags for the random mobility pattern of a robot. For each experiment, the average localization error is noted down and the threshold that gave lowest localization error is chosen as the lower bound on the number of tags for the given deployment scenario. Here, we take advantage of the fact that the RFID tags deployment is going to be static and thus the measurement incurs only a one-time cost. 


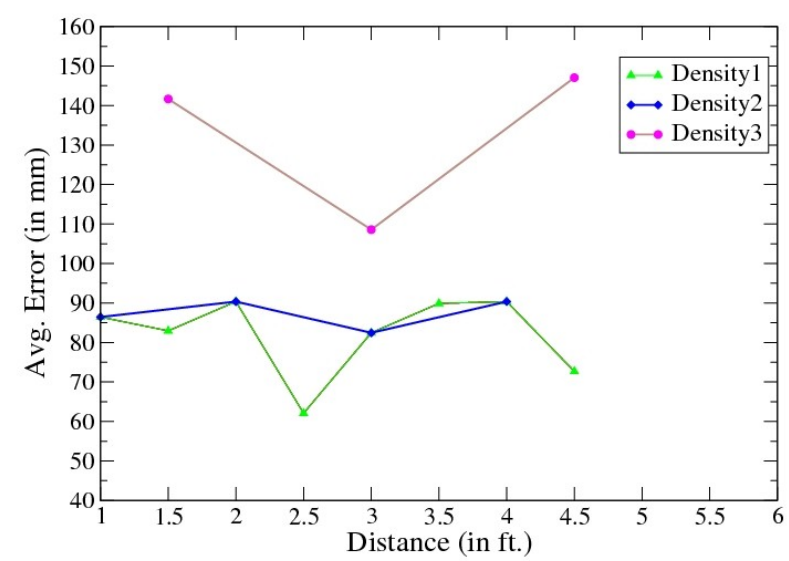

Fig. 4. Measurements based study of ALS to determine the lower bound on the number of RFID tags to be used. Here, number of tags is mapped to the distance between the first and the last tag, in order to normalize across the readings for multiple densities.

\section{EXPERIMENTAL EVALUATION}

This section presents an evaluation of RCRA and ALS based localization. Specifically, we first evaluate ALS for different densities of RFID tags deployment to determine the lower bound for the number of RFID tags to be considered. We then incorporate this information in the ALS, and compare its performance against the other policies, namely the pure sensors based readings, two-tags based calibration (original approach), and RCRA.

\section{A. Study of ALS for Different Tags Densities}

As discussed before, the angle and distance sensors mounted on iRobot Create are not sufficient in themselves to accurately track the position of the robot in real-time. Thus, we rely on an RFID-based external mechanism to improve the localization accuracy of robots. Although, detection of an RFID tag enables position calibration of the robot with a fixed maximum error margin, the robot has to wait until it crosses the second RFID tag in a straight line in order to determine its orientation. Since, the RFID tag's reading range is not a point, but a circle of fixed radius, orientation calculation using two tags can have large error margin compared to the pure sensors based orientation, depending on the distance between those tags. This error margin reduces as the number of tags encountered on a straight line increases. Thus, the goal of this experiment is to study this tradeoff, and determine the average number of tags that should be crossed by the robot in order to use the RFID-based calibration. We perform this experiment for three different densities of RFID tags deployment. Essentially, we deploy the tags uniformly such that the horizontal and vertical distances between pairs of tags is varied to create different densities.

In order to conduct the experiment, we run the robot with a random mobility pattern over the testbed of deployed

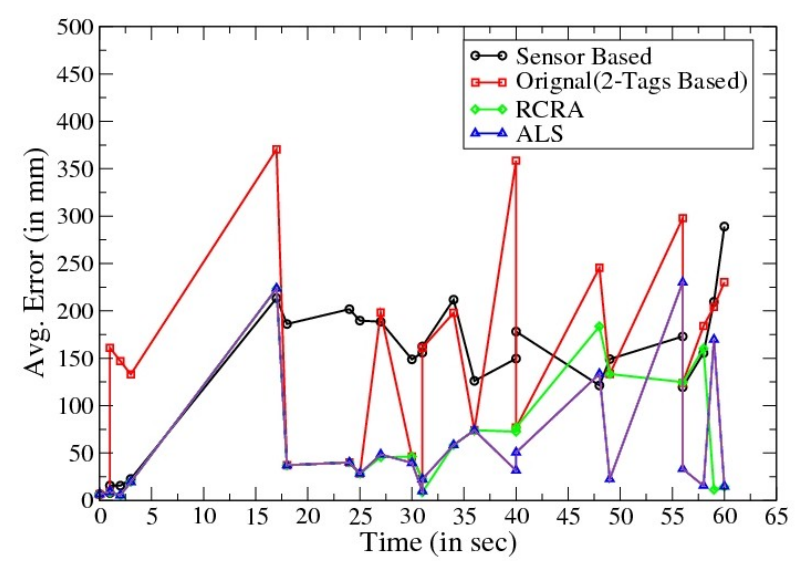

Fig. 5. Comparison of different policies for Densityl.

RFID tags. Once the robot is initialized with its position and orientation (after the initialization process), it keeps track of the position estimates generated by the RFID-based calibrated readings with continuous refinement enabled, while it is mobile. When the robot encounters an RFID tag, we note down the error for the RFID-based calibration, which we average out at the end of the robot's mobility script. We conduct this experiment for different values of the lower bound on the number of RFID tags for the ALS, and chose the value of the lower bound on the number of RFID tags that results in the smallest average localization error. Figure 4 shows the average localization error observed for the ALS, for experiments conducted over different RFID tags densities. Please note that, the experiments were conducted for different values of the lower bound of the number of RFID tags, but in order to normalize the readings across multiple tags densities, we used distance as a metric, instead. For this experiment, tags are deployed uniformly with the horizontal and vertical distances between pairs of tags set to $0.5 \mathrm{ft}$. for Densityl, $1.0 \mathrm{ft}$. for Density2, and 1.5 ft. for Density3.

As the graph indicates, the smallest error was observed when the RFID-based orientation calibration was performed using tags that were spaced apart with distances close to $2.5 \mathrm{ft}$ or $3 f t$, across all the tags densities. Thus, we can conclude that if the robot crosses two RFID-tags that are spaced apart by at least $3 \mathrm{ft}$. distance, the robot can use RFID-based calibration to calibrate its orientation, while it can fall back to its sensors based orientation value if the distance between tags is less than $3 f t$.. Here, $3 f t$. of distance constitutes 6,3 , and 2 RFID tags for Density1, Density2, and Density3, respectively.

In the next section, we present comparison of the different localization policies, and we configure ALS to use the value of the lower bound on the number of tags that need to be detected over a straight line movement obtained through this study. 


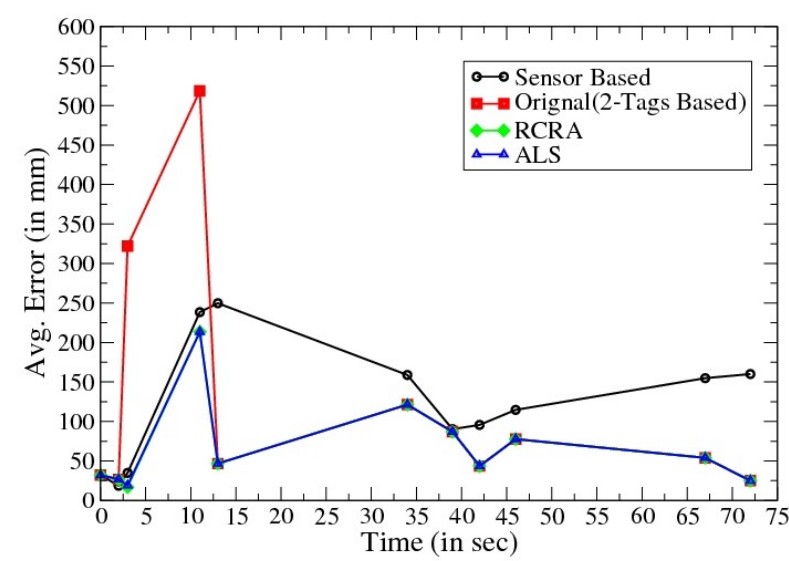

Fig. 6. Comparison of different policies for Density2.

\begin{tabular}{|c|c|c|c|c|c|}
\hline Density & $\begin{array}{c}\text { Inter-tag } \\
\text { distance } \\
\text { in ft. }\end{array}$ & $\begin{array}{c}\text { Sensors } \\
\text { based } \\
\text { in } \mathrm{mm} .\end{array}$ & $\begin{array}{c}\text { Original } \\
\text { 2-tags based } \\
\text { in } \mathrm{mm} .\end{array}$ & RCRA & ALS \\
\hline Density1 & 0.5 & 148.59 & 147.2 & 70.33 & in $\mathrm{mm}$. \\
\hline Density2 & 1.0 & 130.28 & 143.92 & 73.56 & 82.08 \\
\hline Density3 & 1.5 & 100.05 & 108.57 & 108.57 & 108.57 \\
\hline
\end{tabular}

TABLE I

AVERAGE LOCALIZATION ERRORS FOR DIFFERENT POLICIES.

\section{B. Comparison of Different Localization Policies}

The goal of this experiment is to compare the performance of different localization policies. As mentioned before, the experiment is conducted for three different densities of uniformly deployed RFID tags. We move the robot in random fashion over the testbed area of deployed RFID tags, after the initialization process, and keep track of the positions estimated by different localization policies: (1) Sensors-based: position is updated solely based on the values of the angle sensor and the distance sensor, (2) Original (2-tags based): this scheme was used in our previous work [7], in which the robot calibrates its position by assigning the coordinates of the RFID tag to itself, and orientation, when it crosses two RFID tags in a straight line, (3) RCRA: it differs from the previous in its orientation calculation procedure as explained in Section III, and (4) $A L S$ : it selects between the sensors-based and RCRA-based orientation value, depending on the lower bound on the number of RFID tags to be used for calibration, as obtained in the previous experiment. We keep track of the estimated positions obtained from each policy, and use their instantaneous value at the time of an RFID tag detection to calculate their errors against the coordinate of the RFID tag. Figures 5, 6, 7 show the comparison of the four localization policies for different RFID tags densities.

As it can be observed from the figures, the performance of Original policy is mainly dependent on the density of RFID tags deployment, and thus for larger densities, it performs

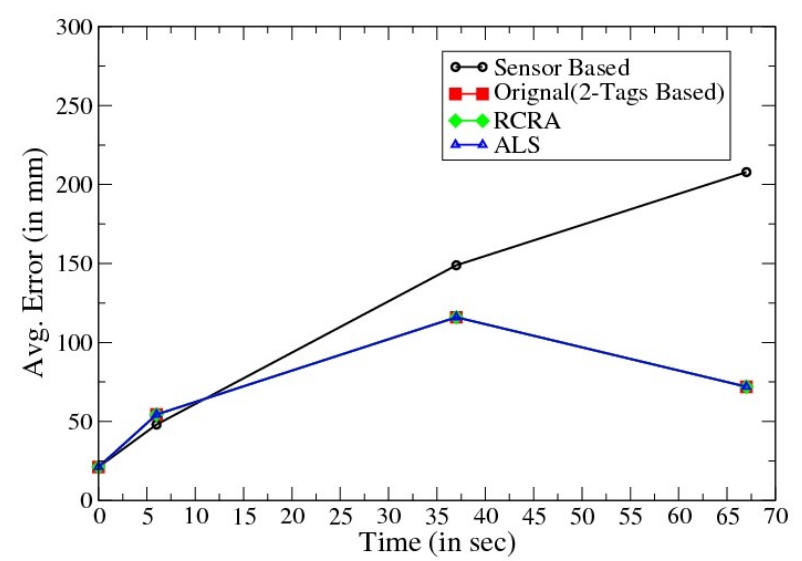

Fig. 7. Comparison of different policies for Density3.

poorly even compared to the pure Sensors based approach. In comparison, $R C R A$ and $A L S$ give much better accuracy most of the times. We can also notice that $A L S$ outperforms RCRA when the RFID tags deployment is dense. However, as the density of RFID tags becomes sparse, all the RFIDbased localization schemes nearly give the same accuracy level. Table I presents localization error values observed for all the policies over different RFID tags densities, averaged across multiple tries. As it can be observed from Table I, the average localization error for $R C R A$ and $A L S$ increases as the density of tags becomes sparser. Thus, $A L S$ for dense deployment of RFID tags appears to give the best performance among the four localization policies used in this work.

\section{RELATED WORK}

The goal of localization is to allow a node to accurately determine its own absolute position and orientation as well as understand positions of the other robots in the testbed. The Roomba robots used in the original MiNT [9], [10] prototype could not sense their own positions. As a result, they relied exclusively on a central controller which used a vision-based position/orientation tracking system consisting of six ceilingmounted webcams with overlapping image planes. This system was used to track node location and to command the robots on which direction to move at any instant. To account for growing discrepancy over time between the central controller's per-node position information and the actual node positions, mobile robots were periodically manually brought to fixed locations to re-synchronize their logical and physical node positions. Each node was identified using unique color patches mounted on the mobile nodes. Since this system relied on visual identification, it tended to develop inaccuracies over time if any of the six cameras moved slightly, or if the color patterns on the nodes faded or lighting varied.

We researched and designed a completely new localization mechanism for the MiNT-2 testbed to overcome the limitations 
of its predecessor. Our system uses inexpensive RFID technology coupled with enhanced mobility sensors within the Create robot to reduce maintenance overhead and simultaneously achieve high levels of accuracy. Since the RFID tags are distributed within the testbed area, a node can localize itself whenever it crosses an RFID tag by assigning the position of the tag to itself, and uses the proposed algorithm to determine its orientation from its movement and RFID history.

Extensive research has been performed [11] on localization in the context of ubiquitous computing, location-aware services and robotics research, which we examined in choosing an appropriate system for MiNT-2 . The focus of the MiNT-2 and MiNT testbeds has been on developing a simple, inexpensive, and practical localization technique for robots used in wireless protocol development and testing context. For our MiNT-2 testbed we considered active and passive infrared triangulation, as well as an infrared version of Stony Brook's MiNT vision system that would use patterns of infrared LEDs instead of colored cards to identify each robot and determine its location and heading. Mobile Emulab [12] also uses a vision-based image processing technique for localization, using two color pattern on top of each robot. In addition to sharing the same drawbacks as the original MiNT testbed, the two color patterns cannot uniquely identify the robots. MIT's Cricket ultrasonic localization system [13] was also considered. However the cost of each module combined with the significant associated computation burden rendered it unsuitable for our application. A pressure-based system called SmartFloor [14] would have been unable to differentiate between the unique nodes, although it is well-suited to determine the precise location of each (anonymous) node. MiNT-2 nodes do not encounter these problems because an inexpensive RFID reader is mounted on each robot and thus the node itself can detect/compute its location without relying on any external data. RFID based localization techniques have also proven effective in other contexts for both large-scale localization [15] and more limited table-sized applications [16].

\section{CONCLUSION AND FUtURE WORK}

In this paper, we address the sources of inaccuracy associated with the RFID-based localization approach proposed in our previous work, MiNT-2. Although, RFID-based calibration helped assigning correct position to the robot upon detection of an RFID tag, the location error observed between two successive tags detections was significant, mainly because of the large error margin associated with the robot's orientation calibration mechanism. In order to address this limitation, we proposed RCRA, a continuous orientation-refinement based approach, and ALS, an hybrid approach which adapts between sensors-based values and the RCRA to get better orientation accuracy. Through our experimental evaluation, we show that the proposed approaches improve the accuracy of RFIDbased localization mechanisms by up to $58.22 \%$ for dense deployment of RFID tags.

The current mechanism to determine the lower bound on the number of RFID tags required for orientation calibration in ALS is static - based on the measurements. In future, we plan to extend the ALS to dynamically determine the lowerbound on minimum number of RFID tags required, in order to make the scheme more generic.

\section{REFERENCES}

[1] Information Sciences Institute, "The Network Simulator - NS-2 http://www.isi.edu/nsnam/ns/."

[2] X. Zeng, R. Bagrodia, and M. Gerla, "GloMoSim: A Library for Parallel Simulation of Large-Scale Wireless Networks," in Workshop on Parallel and Distributed Simulation, May 1998.

[3] Scalable Network Technologies Inc., "QualNet WiFi simulator," in http://www.scalable-networks.com/products/qualnet_wifi.php, 2004.

[4] D. Maltz, J. Broch, and D. Johnson, "Experiences Designing and Building a Multi-Hop Wireless Ad-Hoc Network Testbed," in Technical Report 99-116, School of Computer Science, CMU, Mar 1999.

[5] H. Lunndgren, D. Lundberg, J. Nielsen, E. Nordstrom, and C. Tscudin, "A Large-scale Testbed for Reproducible Ad Hoc Protocol Evaluations," in Proceedings of Wireless Communications and Networking (WCNC), 2002.

[6] Daniel Aguayo et al., "MIT Roofnet Implementation http://www.pdos.lcs.mit.edu/roofnet/design/," August 2003.

[7] C. Mitchell, V. Munishwar, S. Singh, X. Wang, K. Gopalan, and N. AbuGhazaleh, "Testbed design and localization in mint-2: A miniaturized robotic platform for wireless protocol development and emulation," in Proc. First International Conference on Communication systems and Networks (COMSNETS), Jan. 2009.

[8] JFW Industries, Inc., "Fixed Attenuators http://www.jfwindustries.com/Cat2000/FixedAttenuatorsTerminations.pdf."

[9] P. De, R. Krishnan, A. Raniwala, K. Tatavarthi, N. A. Syed, J. Modi, and T. cker Chiueh, "Mint-m: An autonomous mobile wireless experimentation platform," In Proceedings of Mobisys, 2006.

[10] P. De, A. Raniwala, S. Sharma, and T. cker Chiueh, "Mint: A miniaturized network testbed for mobile wireless research," In Proceedings of IEEE Infocom, 2005.

[11] J. Hightower and G. Borriello, "Location systems for ubiquitous computing," Computer, vol. 34, no. 8, pp. 57-66, Aug 2001.

[12] B. White, J. Lepreau, L. Stoller, R. Ricci, S. Guruprasad, M. Newbold, M. Hibler, C. Barb, and A. Joglekar, "An integrated experimental environment for distributed systems and networks," in Proc of OSDI'02, Boston, MA, Dec. 2002, pp. 255-270.

[13] N. B. Priyantha, A. Chakraborty, and H. Balakrishnan, "The cricket location-support system," in MobiCom '00: Proceedings of the 6th annual international conference on Mobile computing and networking. New York, NY, USA: ACM, 2000, pp. 32-43.

[14] R. J. Orr, "SmartFloor, http://www.cc.gatech.edu/fce/smartfloor/."

[15] J. Bohn, "Prototypical implementation of location-aware services based on a middleware architecture for super-distributed rfid tag infrastructures," Personal Ubiquitous Comput., vol. 12, no. 2, pp. 155-166, 2008.

[16] S. Hinske and M. Langheinrich, "An RFID-based Infrastructure for Automatically Determining the Position and Orientation of Game Objects in Tabletop Games." 